\title{
OIL SPILL AISA+ HYPERSPECTRAL DATA DETECTION BASED ON DIFFERENT SEA SURFACE GLINT SUPPRESSION METHODS
}

\author{
Yang Junfang ${ }^{1,2}$, Ren Guangbo ${ }^{2}$, Ma Yi $^{2 *}$, Dong Liang ${ }^{3}$, Wan Jianhua ${ }^{1}$ \\ ${ }^{1}$ School of Geosciences, China University of Petroleum, Qingdao 266061, China, yangjunfang113@163.com, \\ ${ }^{2}$ The First Institute of Oceanography, SOA, Qingdao 266061, China, mayimail@ fio.org.cn, renguangbo@126.com \\ ${ }^{3}$ North China Sea Branch, SOA, Qingdao 266061, China, dongliang@bhfj.gov.cn
}

\author{
Commission III, WG III/4
}

KEY WORDS: Oil Spill Detection, Hyperspectral Data, Glint Suppression, Wavelet Transform, Enhanced Lee Filter

\begin{abstract}
:
The marine oil spill is a sudden event, and the airborne hyperspectral means to detect the oil spill is an important part of the rapid response. Sun glint, the specular reflection of sun light from water surface to sensor, is inevitable due to the limitation of observation geometry, which makes so much bright glint in image that it is difficult to extract oil spill feature information from the remote sensing data. This paper takes AISA+ airborne hyperspectral oil spill image as data source, using multi-scale wavelet transform, enhanced Lee filter, enhanced Frost filter and mean filter method for sea surface glint suppression of images. And then the classical SVM method is used for the oil spill information detection, and oil spill information distribution map obtained by human-computer interactive interpretation is used to verify the accuracy of oil spill detection. The results show that the above methods can effectively suppress the sea surface glints and improve the accuracy of oil spill detection. The enhanced Lee filter method has the highest detection accuracy of $88.28 \%$, which is $12.2 \%$ higher than that of the original image.
\end{abstract}

\section{INTRODUCTION}

With the development of maritime transport industry, the accidents of oil spill frequently occur at sea. Oil spill is a serious marine accident that pollute the marine ecological environment. In June 2011, a serious oil spill accident happened in Penglai 19-3 oil field located in China Bohai Sea, which belongs to ConocoPhillips China. The total oil spill volume reached more than 7 thousand tons, and the polluted sea area was about 6200 square kilometers. The accident caused serious harm to the marine ecological environment and biological resources of Bohai Sea, causing great losses to fishermen in three provinces and one city such as Hebei, Liaoning, Shandong and Tianjin. The compensation for oil spill accident achieved 1.683 billion Yuan.

Hyperspectral image has the characteristics of numerous bands, high resolution, abundant information. Compared with the multispectral image, the detail information of hyperspectral image is especially outstanding, which can better distinguish the difference between seawater and oil film. It is beneficial to obtain more realistic and objective oil spill data, and to improve the ability of detecting and recognizing oil spill through the subdivision of spectral bands( $\mathrm{Li}, 1996)$. Marine oil spill is usually far away from the mainland. Timely information is fundamental to reduce the impact of oil spill (Casciello et al., 2011). Aviation and satellite remote sensing are effective means for monitoring this area. Airborne hyperspectral can react quickly to emergencies and has incomparable advantages in finding oil spills in time. In general, the sea surface can be regarded as an undulating surface composed of numerous smooth small surfaces, and a specular reflection will occur when the sunlight reaches the sea surface (Chen et al., 2009; Mao et al., 1996). For airborne remote sensing, due to the limitation of observation geometry, the sun glint entering the sensor are very severe and inevitable when the airplane head for the direction of the sun, which makes so much bright glint in image that it is difficult to extract oil spill feature information from the remote sensing data (Mustard et al., 2001) Therefore, methods of sea surface glint suppression for image processing should be paid more attention. At present, many studies have established the corresponding relationship between the visible and near infrared bands through regression analysis to eliminate sea surface glint (Goodman et al., 2008; Hedlry et al., 2005; Hochberg et al., 2003; Kutser et al., 2009; Lyzenga et al., 2006). However, studies on sea surface glint suppression for airborne hyperspectral data are rare.

This paper takes AISA+ airborne hyperspectral oil spill image as data source, using multi-scale wavelet transform, enhanced Lee filter, enhanced Frost filter and mean filter method for sea surface glint suppression of images. And then the classical SVM method is used for the oil spill information detection, and oil spill information distribution map obtained by humancomputer interactive interpretation is used to verify the accuracy of oil spill detection.

\section{DATA AND METHOD}

\subsection{Data}

The AISA + airborne hyperspectral oil spill image used in this paper was acquired by China Marine Surveillance North Sea Aviation detachment on August 23, 2011 in the Penglai 19-3 C platform (Figure 1). The AISA + image has 258 bands, its wavelength of spectral bands range from $400 \mathrm{~nm}$ to $970 \mathrm{~nm}$, and spectral resolution is $2.9 \mathrm{~nm}$, therefore, it has the ability to distinguish differences in characteristics of sea water and oil

* Corresponding author: Yi MA, E-mail: mayimail@fio.org.cn 
film. In order to validate the accuracy of oil spill detection, the field aerial photo and the oil spill interpretation map obtained by human-computer interactive method is used in this paper for validation (Figure 2).

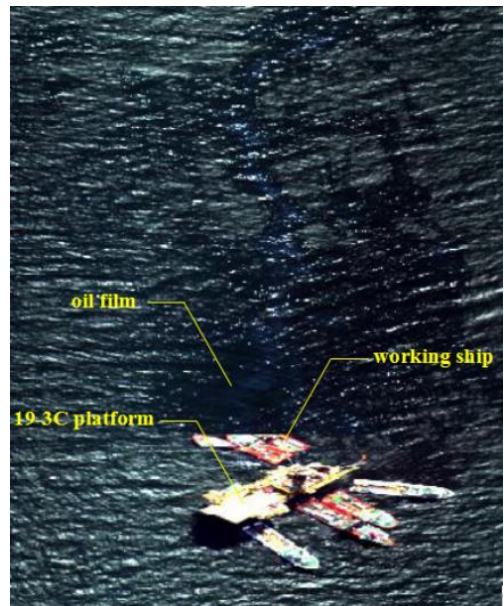

Figure 1. AISA+ hyperspectral image（R 107, G 68, B 28)

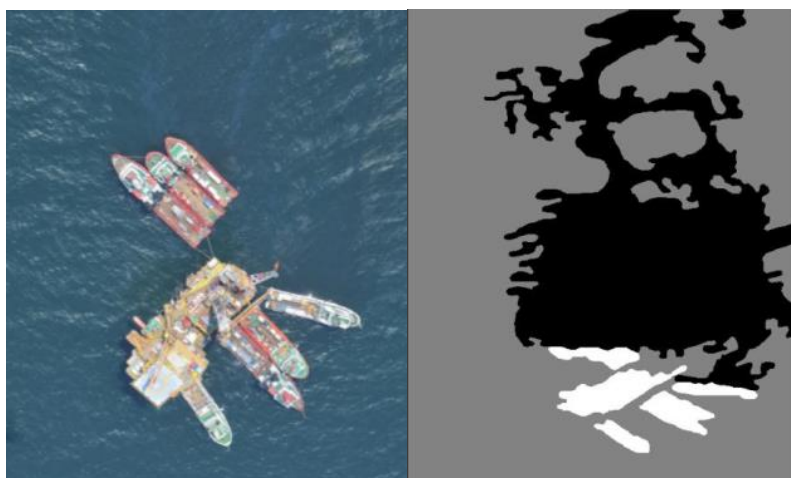

Figure 2. The field aerial photo and validation map (black is oil film, gray is seawater, and white is working ship and platform)

\subsection{Methods for Glint Suppression}

The brightness of the sun glint shows a regional change in the image. In order to eliminate the influence of the sun glint on the detection of the oil spill information. This paper used sea surface glint suppression methods (Figure 3), such as the multiscale wavelet transform, enhanced Lee filter, enhanced Frost filter and mean filter, to process sun glint noise in the AISA+ airborne hyperspectral remote sensing image.

Wavelet transform, a new transform analysis method, inherits and develops the idea of short time Fourier transform localization, and overcomes the disadvantage of window size not vary with frequency. Its main feature as follows: (1) Wavelet decomposition can cover the whole frequency domain. (2) It can greatly reduce or remove the correlation between different feature through selecting suitable filter. (3) Wavelet transform has the "zoom" feature, which can fully highlight the characteristics of some aspects of the problem through the transformation, and can analyze locally the time (space) frequency. The signal (function) is gradually multi-scale refined by the telescopic translation operation so that any detail of the signal can be focused. The processed images of 1-level, 2-level, 3-level and 4-level wavelet transform are shown in Figure 3(a)-(d).
Enhanced Lee filter is a typical method of image speckle filtering based on local statistical characteristics of images. Its principle is based on the fully developed speckle noise model, selecting a certain size window as a local area, calculating its mean and variance so as to achieve the effect of image denoising. The mathematical expression of the filter is as follows:

$$
R=\left\{\begin{array}{ll}
\bar{g}_{i j} & \sigma_{i j} \leq c_{u} \\
\bar{g}_{i j} w+\mathrm{g}(1-w) & c_{u}<\sigma_{i j}<c_{\max } \\
g_{i j} & \sigma_{i j} \geq c_{\max }
\end{array}\right\}
$$

Where $R=$ pixel gray value after denoise

$w=$ weighting function

$\sigma_{i j}=$ local standard deviation

$g_{i j}, \bar{g}_{i j}=$ original gray value, average gray value of each pixel in the calculation window, respectively

$$
c_{u}, c_{\max }=\text { threshold }
$$

Enhanced Frost filter, a equalization filter, circulates with damping index based on local statistical characteristics of images, which can denoise according to the variance coefficient calculated in the filter window, and it can better maintain details in the image. The mathematical expression of the filter is as follows:

$$
R=\left\{\begin{array}{ll}
\bar{g}_{i j} & c_{l}<c_{u} \\
\sum_{i=1}^{n} \sum_{j=1}^{n} g_{i j} \times M_{i j} / \sum_{i=1}^{n} \sum_{j=1}^{n} M_{i j} & \\
g_{i j} & c_{u} \leq c_{l} \leq c_{\max }>c_{\max }
\end{array}\right\}
$$

Where $R$ = pixel gray value after denoise

$M_{i j}=$ weight index of each corresponding pixel

$g_{i j}, \bar{g}_{i j}=$ original gray value, average gray value of each pixel in the calculation window, respectively

$c_{l}=\sigma_{i j} / \bar{g}_{i j} ; c_{u}=1 / \sqrt{L} ; c_{\max }=\sqrt{1+2 / L}$

$L=$ imaging number of looks

Median filter is a nonlinear processing method to remove noise, which assumes that the signal has an extreme value, that is, the noise is a maximum or a minimum in a smooth window. Median filter takes the median of all pixels in the smooth region as the center pixel value of the smooth region. It can preserve the edge information of the image while denoising. The mathematical expression of the filter is as follows:

$$
R=\operatorname{med}\left(D N_{i j}^{\prime}\right)
$$

Where $R=$ gray value of central pixel after denoise 
The International Archives of the Photogrammetry, Remote Sensing and Spatial Information Sciences, Volume XLII-3, 2018 ISPRS TC III Mid-term Symposium "Developments, Technologies and Applications in Remote Sensing", 7-10 May, Beijing, China

$D N_{i j}^{\prime}=$ gray value of each pixel after rearrangement in the smooth window
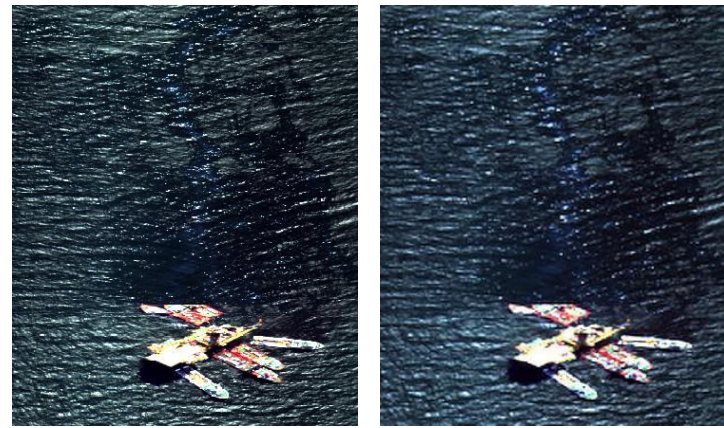

(a) original image

(b) 1-level wavelet transform
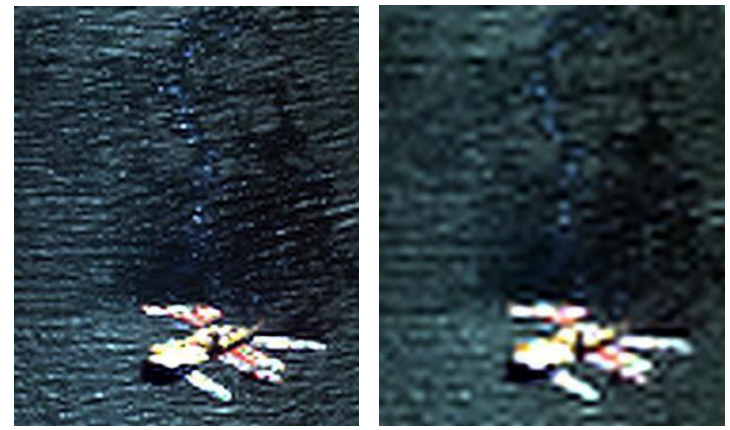

(c) 2-level wavelet transform（d) 3-level wavelet transform
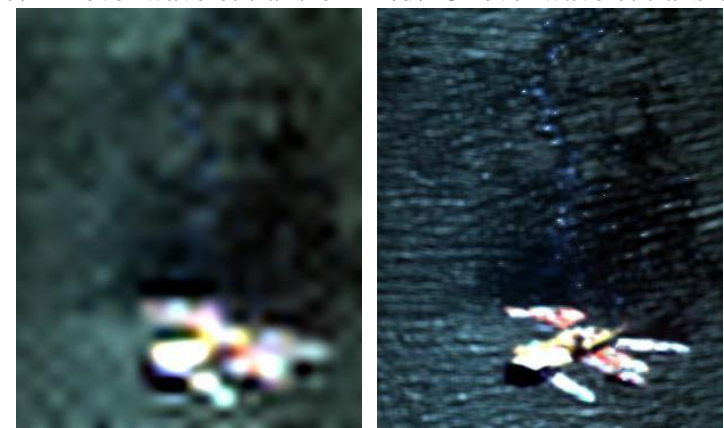

(e) 4-level wavelet transform（f) enhanced Lee filter

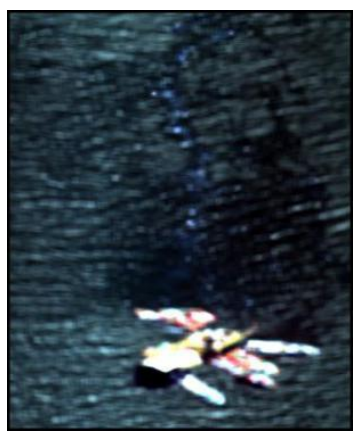

(g) enhanced Frost filter

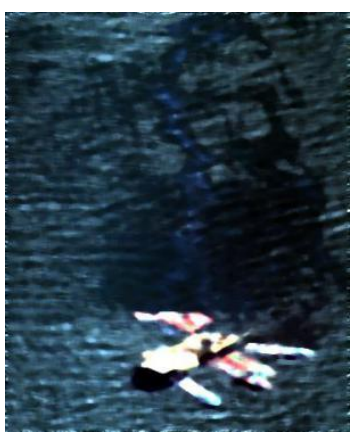

(h) Median filter
Figure 3. Different methods of sun glint suppression

\subsection{Classification Method}

SVM, an excellent classification method, has two main characteristics: (1) SVM is well applied for linear separation situation. For other' s situation, SVM transform lowdimension input space and linear inseparable sample into high dimensional feature space by using nonlinear algorithm. (2) Based on Structural Risk Minimization Theory, SVM constructs optimal segmentation hyperplane in the feature space.

\section{RESULTS AND ANALYSIS}

\subsection{Detection Results}

The above methods are used to suppress the sun glint, and the oil spill information detection is conducted by the classical SVM method. The detection results are shown in Figure 4.
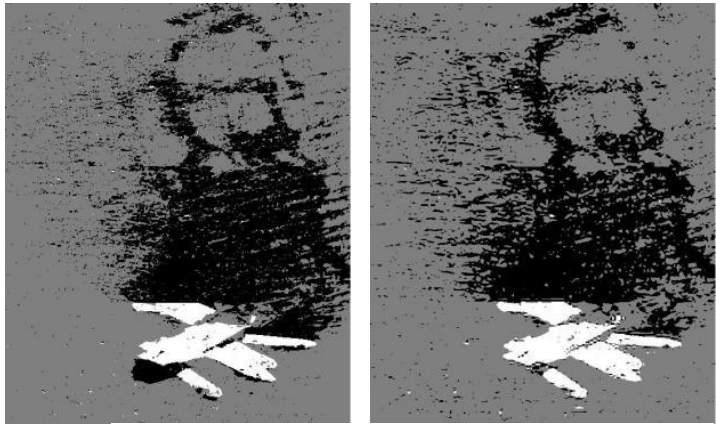

(a) original image

b) 1-level wavelet transform
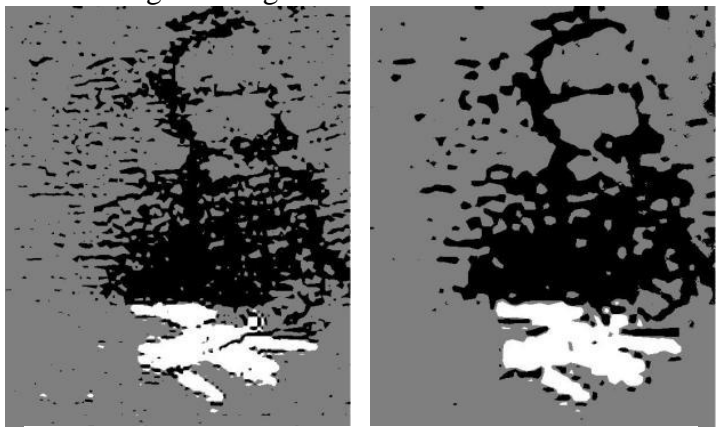

(c) 2-level wavelet transform (d) 3-level wavelet

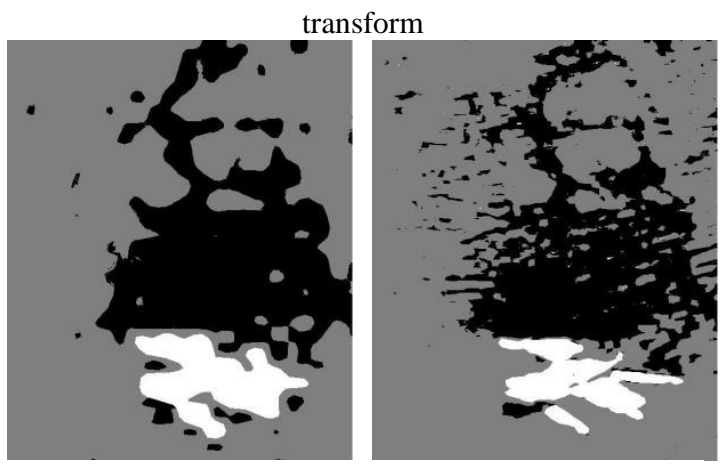

(e) 4-level wavelet transform（f) enhanced Lee filter

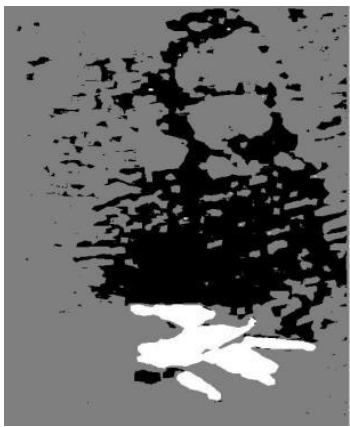

(g) enhanced Frost filter

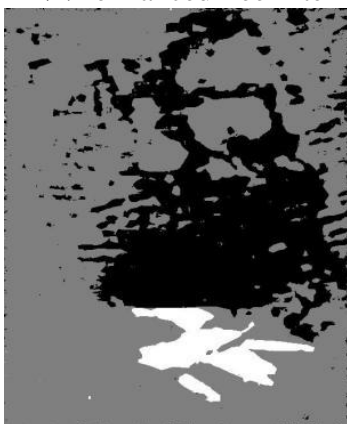

(h) Median filter
Figure 4. SVM detection results of different sun glint suppression method (black is oil film, gray is seawater, and white is working ship and platform ) 


\subsection{Accuracy Evaluation}

Based on statistical results(Table 1) of the confusion matrix, using three indicators to evaluate the accuracy: The classification accuracy for all types was evaluated by the overall classification accuracy and kappa coefficient ; the classification accuracy for each type of feature was evaluated by producer accuracy .

\begin{tabular}{ccccccccc}
\hline Types & $\begin{array}{c}\text { Original } \\
\text { image }\end{array}$ & $\begin{array}{c}\text { 1-level } \\
\text { wavelet }\end{array}$ & $\begin{array}{c}\text { 2-level } \\
\text { wavelet }\end{array}$ & $\begin{array}{c}\text { 3-level } \\
\text { wavelet }\end{array}$ & $\begin{array}{c}\text { 4-level } \\
\text { wavelet }\end{array}$ & $\begin{array}{c}\text { Enhanced } \\
\text { Frost filter }\end{array}$ & $\begin{array}{c}\text { Enhanced } \\
\text { Lee filter }\end{array}$ & $\begin{array}{c}\text { Median } \\
\text { filter }\end{array}$ \\
\hline Oil film & 76.08 & 77.21 & 79.11 & 82.88 & 81.48 & 86.64 & 88.28 & 84.35 \\
Water & 90.07 & 90.47 & 90.35 & 89.06 & 89.91 & 92.73 & 92.39 & 91.90 \\
Ship and platform & 89.23 & 91.87 & 92.09 & 92.16 & 90.27 & 92.93 & 94.35 & 92.82 \\
Overall accuracy & 84.69 & 85.75 & 86.80 & 88.96 & 87.32 & 90.62 & 92.78 & 89.98 \\
Kappa & 0.7371 & 0.7377 & 0.7406 & 0.7775 & 0.7525 & 0.8401 & 0.8514 & 0.8080 \\
\hline
\end{tabular}

Table 1. SVM detection accuracy of different flare suppression method (\%)

Table 1 shows the accuracy of SVM method for oil spill detection based on the original AISA+ airborne hyperspectral remote sensing image is $76.08 \%$, Kappa coefficient is 0.7371 , and oil spill detection accuracy are improved after sea surface glint suppression. Among them, enhanced Lee filter method has the highest accuracy of $88.28 \%$, which is $12.2 \%$ higher than that of the original image, the reason is that enhanced Lee filter is constructed according to the principle of different filters were applied in different regions of the image. For homogeneous regions, the noise can be smoothed simply with mean filter. For heterogeneous regions, Lee filters applied to preserve texture information when noise is removed. At the same time, enhanced Lee filter method has the highest Kappa coefficient of 0.8514 , which is 0.1143 higher than that of the original image, it shows that the evaluators have good consistency and the classification results are better, which reflects that the Lee filter method can effectively suppress sea surface glint. It is followed by enhanced Frost filter method and median filter method, with oil spill detection accuracy of $86.64 \%$ and $84.35 \%$, respectively. Because of the shadow of the platform is identified as an oil film by both of two methods, the detection accuracy of the two is lower than that of the enhanced Lee filter. For the different scale wavelet transform, the 3-level wavelet transform has the highest oil spill detection accuracy, reaching $82.88 \%$, which is $5.67 \%$ higher than that of the 1 - level wavelet transform. With the increase of the wavelet transform level, the detection accuracy first increases and then decreases. This is because the edge details of the working ship and platform are blurred. In the 4 level wavelet transform, the image quality is drop obviously, and the details information is lost.

\section{CONCLUSIONS}

Oil spill is a serious marine accident that pollute the marine ecological environment. Sun glint is inevitable due to the limitation of observation geometry, which makes so much bright glint in image that it is difficult to extract oil spill feature information from the remote sensing data. This paper takes AISA+ airborne hyperspectral oil spill image as data source, using multi-scale wavelet transform, enhanced Lee filter, enhanced Frost filter and mean filter method for sea surface glint suppression of images. And then the classical SVM method is used for the oil spill information detection, and oil spill information distribution map obtained by humancomputer interactive interpretation is used to verify the accuracy of oil spill detection. The accuracy of SVM method for oil spill detection based on the original AISA+ airborne hyperspectral remote sensing image is $76.08 \%$, Kappa coefficient is 0.7371 . The enhanced Lee filter method has the highest detection accuracy of $88.28 \%$, which is $12.2 \%$ higher than that of the original image. It is followed by enhanced Frost filter method and median filter method, with oil spill detection accuracy of $86.64 \%$ and $84.35 \%$, respectively. For the different scale wavelet transform, the 3-level wavelet transform has the highest oil spill detection accuracy, reaching $82.88 \%$, which is $5.67 \%$ higher than that of the 1- level wavelet transform. The results indicate that the sea surface glint in the image seriously affects the oil spill detection. The above method can effectively suppress the sun glint information and improve the accuracy of oil spill hyperspectral detection.

\section{ACKNOWLEDGMENTS}

This study is fully funded by National Natural Science Foundation of China (No. 41206172) and the National Natural Science Foundation of China (No. 61601133).

I'd like to express my sincere appreciation to China Marine Surveillance North Sea Aviation detachment for their efforts in providing the AISA + airborne hyperspectral oil spill image.

\section{REFERENCES}

Chen L., Deng R. R., Xie J., 2009. Research on oil spill monitoring in offshore oil development based on AISA+ Airborne Hyperspectral. Transactions of Oceanology and Limnology, 1, pp. 179-184.

GOODMAN J A., LEE Z., USTIN S. L. 2008. Influence of atmospheric and sea-surface corrections on retrieval of bottom depth and reflectance using a semi-analytical model: A case study in Kaneohe bay, Hawaii. Applied Optics, 47 (28), pp. F1-F11.

HEDLRY J., HARBORNE A., MUMBY P., 2005. Simple and robust removal of Sun glint for mapping shallow-water benthos. International Journal of Remote Sensing, 26(10), pp. 2107-2112.

HOCHBERG E., ANDREFOUET S. TYLER M., 2003. Sea surface correction of high spatial resolution Ikonos images to improve bottom mapping in near-shore environments. IEEE Transactions on Geoscience and Remote Sensing, 41(7), pp. 1724-1729.

KUTSER T., VAHTMAE E., PRAKS J., 2009. A Sun glint correction method for hyperspectral imagery containing areas 
with nonnegligible water leaving NIR signal. Remote Sensing of Environment, 113(10) , pp. 2267-2274.

Li, S. H., 2004. Remote sensing detection technology and application progress of offshore oil spill. Remote Sensing Information, 2, pp. 53-57.

LYZENGA D., MALINAS N., TANIS F., 2006. Multispectral bathymetry using a simple physically based algorithm.

IEEE Transactions on Geoscience and Remote Sensing, 44 (8) , pp. 2251-2259.

Mao, Z. H., Guo D. F., 1996. Study on the extraction of remote sensing aviation color information and elimination method of sun glint. Remote Sensing Technology and Application,11(4), pp. $15-20$.

Mustard, J. F., Staid, M. I., \& Fripp, W. J., 2001. A semianalytical approach to the calibration of AVIRIS data to reflectance over water application in a temperate estuary. Remote Sensing of Environment, 75, pp. 335-349.

Casciello, D., Lacava T., Pergola N. \& Tramutoli V., 2011. Robust satellite techniques for oil spill detection and monitoring using AVHRR thermal infrared bands, International Journal of Remote Sensing, 32(14), pp. 41074129. 